\title{
Promover as tecnologias de informação geográfica na formação para a docência
}

\author{
Luísa Azevedo ${ }^{1}$, Vítor Ribeiro ${ }^{2}$, António Osório ${ }^{3}$ \\ Luisa_9576@hotmail.com, vitor.geografia@gmail.com, ajosorio@ie.uminho.pt \\ ${ }^{1}$ CIEd/Lab2PT, Universidade do Minho, Portugal \\ ${ }^{2}$ Lab2PT, Universidade do Minho, ESEPF, Portugal \\ ${ }^{3}$ CIEd, Universidade do Minho, Portugal
}

\begin{abstract}
Resumo
A evolução tecnológica das últimas décadas requer, às escolas, uma adaptação às mudanças que ocorrem na sociedade. As práticas pedagógicas devem promover a construção do conhecimento e a aprendizagem coletiva, entre professores, alunos e comunidade educativa, recorrendo, nalguns casos, ao uso das Tecnologias de Informação Geográfica (TIG), para desenvolver as competências exigidas à sociedade do século XXI. Neste sentido, as instituições de ensino superior que intervêm na formação de professores têm o importante papel de dotar os futuros docentes com competências digitais que lhes permitam integrar as TIG nas suas práticas pedagógicas. Nesta comunicação pretende-se partilhar a metodologia utilizada no projeto "Atlas de África" desenvolvido em dois mil e dezasseis, por estudantes de Mestrado em Ensino do 1.ำ e do 2.ำ ciclo do ensino básico. A componente principal deste projeto incidiu na utilização das Tecnologias de Informação e Comunicação (TIC) e das TIG numa perspetiva histórica/cultural, económica e geográfica, resultando na publicação de um atlas geográfico do continente africano. De igual modo, pretendeu-se desenvolver as competências geográficas para renovar as práticas pedagógicas, valorizando o pensamento espacial e o raciocínio crítico. Para concretizar este projeto recorreu-se a uma metodologia participativa para promover o trabalho colaborativo e investigativo. Os resultados deste projeto foram muito positivos e estenderam-se para além da sala de aula.
\end{abstract}

Palavras-Chave: TIG; TIC; formação docente; geografia; ensino/aprendizagem.

\begin{abstract}
The technological evolution of the last decades requires, from schools, an adaptation to the changes in our society. Pedagogical practices should promote the building of knowledge by pupils, in some cases, by use of Geographic Information Technologies (GIT) to promote the skills required of the 21st century society. In this sense, higher education institutions, namely those that intervene in teacher training, have the important role of providing future teachers with skills that allow them to integrate GIT into their pedagogical practices. The aim of this paper is to share the methodology used in the project "Atlas of Africa", developed in 2016 by Master's students in the $1^{\text {st }}$ and $2^{\text {nd }}$ cycle of Portuguese Basic Education. The main component of this project was the use of Information and Communication Technologies (ICT) and GIT in a historical/cultural, economic and geographical perspective, resulting in the publication of a geographic atlas of the African continent. Likewise, it was intended to develop geographic skills to renew pedagogical practices, valuing spatial thinking and critical thinking. In order to realize this project, a participatory methodology was used to promote collaborative and investigative work. The results of this project were very positive and extended beyond the classroom.
\end{abstract}

Keywords: GIT; ICT; teacher training; geography; teaching and learning. 


\section{Introdução}

As mudanças ocorridas na sociedade, resultantes da crescente evolução tecnológica a que se tem assistido nas últimas décadas, requer, à escola, uma (re)adaptação do seu papel. Na atual sociedade da informação e do conhecimento, as gerações mais jovens, identificadas por Prensky (2001) como a geração dos nativos digitais contactam e utilizam, diariamente, as tecnologias. Uma vez que estas fazem parte do seu quotidiano, as aprendizagens dos indivíduos devem ser sustentadas em abordagens construtivistas e em ambientes inovadores com recurso às tecnologias. A renuncia ao paradigma mecanicista, que outrora fora utilizado para a massificação da escola, e que ainda hoje caracteriza o nosso sistema de ensino, é uma inevitabilidade. Desenvolver novas práticas recorrendo, nalguns casos, ao uso de tecnologias emergentes, nomeadamente das Tecnologias de Informação Geográfica (TIG), para promover, nas gerações mais jovens, o desenvolvimento das competências exigidas à sociedade do século XXI é premente.

O objetivo primordial das instituições de ensino, hoje, deve ser o de preparar os estudantes para o "mundo real", um desafio cada vez maior e mais complexo, auferido não só à comunidade escolar, mas a toda a comunidade educativa que, (in)diretamente, atua nos diversos contextos, designadamente no desenvolvimento pessoal e social dos indivíduos. É necessário habilitar os sujeitos para um mundo que se encontra em constante mudança, cujo futuro é incerto e desconhecido.

Face a estas transformações novas exigências emergem à profissão de professor, pilar do sistema de ensino, requerendo múltiplas competências heterogéneas e altamente complexas, à qual a formação inicial de professores não pode menosprezar. É necessário refletir sobre o atual e futuro papel dos professores nas escolas, as metodologias empregues e o tipo de recursos utilizados em sala de aula, para qualificar os sujeitos com competências cognitivas, interpessoais e intrapessoais, tornando-os cidadãos intervenientes na sociedade. Cabe às instituições de Ensino superior, designadamente as que intervêm na formação de professores, de dotar os seus estudantes, futuros docentes, com as competências e habilidades, em literacia digital, que lhes permitam integrar as TIG nas suas práticas pedagógicas de forma consciente e pedagogicamente integrada, criando ambientes de aprendizagem inovadores. Deste modo, podem promover a literacia geográfica, o pensamento crítico e a participação ativa, dos estudantes.

$\mathrm{Na}$ sequência de uma formação docente que privilegia o desenvolvimento profissional a partir da investigação, da reflexão crítica, da construção do conhecimento e de aquisição de competências tecnológicas foi desenvolvido, no âmbito da unidade curricular de Didática da Geografia, integrada no ciclo de estudos do primeiro ano de Mestrado em Ensino do 1. ${ }^{\circ}$ e do 2. ${ }^{\circ}$ ciclos do ensino básico da Escola Superior de Educação de Paula Frassinetti, na cidade do Porto, um projeto que consistiu na recolha, análise e tratamento de informação gráfica e cartográfica dos países do continente africano.

Assente numa metodologia participativa e colaborativa, o projeto realizado pelas estudantes tem como objetivos:

- desenvolver competências críticas e reflexivas sobre os recursos tecnológicos emergentes e passíveis de serem utilizados em contexto educativo;

- desenvolver a literacia e competências digitais;

- reconhecer as implicações pedagógicas e curriculares consequentes da utilização destas ferramentas na aprendizagem da Geografia;

- promover a articulação teoria/prática, através da mobilização integrada de conhecimentos científicos e didáticos.

\section{Tecnologias de informação geográfica: que contributos para uma educação em transformação?}

A educação é a chave do futuro e a mudança educacional depende fundamentalmente dos docentes e da sua formação (Lopes, 2013). O papel das instituições de Ensino superior que intervém no processo formativo dos sujeitos, futuros docentes, é fundamental na sua preparação para as mudanças que a tecnologias ocasionam no trabalho, no ensino e na aprendizagem (Alves \& Silva, 2015). Ignorar que a 
sociedade disponibiliza um vasto leque de ferramentas tecnológicas, em particular as Geotecnologias, e manter os paradigmas de ensino tradicionalistas, na atualidade, é, por um lado, desperdiçar a oportunidade de preparar os indivíduos para uma sociedade digital e tecnológica, e, por outro, alimentar a memorização de conceitos, descartando o desenvolvimento de competências investigativas, reflexivas e críticas (Azevedo, 2016) essenciais para o mercado de trabalho atual.

As TIG ou Geotecnologias são recursos tecnológicos que admitem a manipulação de informação geográfica, através de ferramentas de análise SIG. Estes, por sua vez, consistem num software que possibilita a aquisição, a compreensão, a indagação e a interpretação de informações sob múltiplas formas, exibindo relações, padrões e tendências espaciais, contidas em mapas, globos, relatórios ou gráficos (Azevedo, 2016). Estas ferramentas admitem a exploração de informação geográfica em recursos estáticos, como por exemplo os mapas analógicos, em recursos digitais (ex. Google Earth, Google Maps) ou ainda em SIG inseridos na web, repartidos em vários dispositivos, como por exemplo smartphone ou tablet (Schultz, Kerski, \& Patterson 2008; Hong 2014; Erskine, Gregg, \& Karimi, 2016). Estas tecnologias permitem relacionar e incorporar informação oriunda de várias fontes e apresentá-la sob a forma de representação cartográfica, tendo em consideração as opções e os interesses do utilizador (Gomes, 2006).

As TIG têm sido amplamente estudadas nos EUA e na Europa, que para além de identificálas como ferramentas tecnológicas vantajosas para diversas áreas de estudo, reconhece, da mesma forma, o seu potencial no contexto educacional. Embora a sua inclusão na área da educação seja ainda muito reduzida, a sua utilização é mais recorrente no ensino secundário e no ensino superior (Berdnarz, \& Schee, 2006; Ribeiro, Remoaldo, \& Gutiérrez, 2015). Sendo muito pouco aplicadas, em ciclos de estudo mais baixos (Demirci, 2009; Goodchild, 2009), as TIG potenciam a transformação dos contextos formais em laboratórios de aprendizagem, ambientes inovadores, dinâmicos e motivadores que alavancam o desenvolvimento do pensamento geográfico e coadjuvam na dissolução das fronteiras existentes entre os contextos de aprendizagem formal e informal (Bednarz, \& Schee, 2006).

A utilização destas tecnologias, nas práticas pedagógicas docentes, minimiza a atitude passiva dos estudantes e fomenta a ação ativa dos mesmos, tornando-os co-construtores do saber. A dinâmica do "aprender fazendo", cuja aprendizagem está centrada no estudante, não só atrai a sua atenção e motivao para a consecução de novos conhecimentos, como melhora o seu raciocínio, promove aprendizagens verdadeiramente significativas e auxiliam-no na confrontação dos desafios de uma sociedade do século XXI (Healey, 2005; Santos, 2010; Spronken-Smith, \& Walker, 2010). O envolvimento constante dos sujeitos em explorações territoriais e em investigações geográficas, com recurso às TIG, contribui para o desenvolvimento do gosto pela descoberta e para a capacidade de resolução de problemas; estimula a sua aproximação com a realidade (Santos, 2010) bem como a sua interação com a comunidade local (Azevedo, 2016).

A eficácia das TIG em integrar bases de dados em diversas camadas de informação possibilita uma leitura da informação de forma rápida e eficiente, através da observação. Desta forma, os estudantes facilmente desenvolvem análises espaciais, estabelecem relações, identificam padrões espaciais e compreendem fenómenos geográficos (Wickson, Carew, \& Russell; 2006, Bednarz, \& Schee 2006; Van der Schee, 2003; Kopcha, Otumfuor, \& Wang, 2015). Por outro lado, as TIG são importantes ferramentas que viabilizam o desenvolvimento de projetos interdisciplinares, dado que não se restringem exclusivamente ao estudo de uma disciplina, mas envolvem várias disciplinas do currículo, melhorando a aprendizagem dos alunos e a compreensão de conceitos (Azevedo, Ribeiro, \& Osório, 2016).

Apesar dos múltiplos benefícios que as TIG oferecem ao mundo educacional, algumas barreiras sobrepõem-se à sua inclusão nas práticas educativas (Tabela 1). Hong (2014), Kerski (2003) e Höhnle, Schubert e Uphues (2011) referem que a complexidade do software dificulta o manuseamento por parte dos estudantes. Assim como as exigências de licenciamentos, com custos associados, conferem, igualmente, obstáculos na implementação das TIG.

Os avanços técnicos e científicos até então desenvolvidos permitiram amenizar estas barreiras, a partir dos Web mapping em múltiplos dispositivos tecnológicos, tais como os smartphones, os tablet ou PDA, que suportam as TIG e tornam-nas mais fáceis de manipular.

Por outro lado, Antunes (2007) aponta que o desconhecimento destas ferramentas por parte dos docentes e do potencial que oferecem no contexto educativo são fatores inibidores da sua utilização 
Tabela 1: Principais barreiras na utilização das TIG.

\begin{tabular}{ll}
\hline Autores & Perspetivas \\
\hline Hong (2014) & Falta de formação docente. \\
& Dificuldade dos alunos no manuseamento das ferramentas. \\
& Falta de tempo no currículo para desenvolver e consolidar a prática de utili- \\
& zação das TIG. \\
\hline Höhnle, Schubert, \& Uphues Falta de formação e prática regular de manuseamento dos SIG. \\
Falta de equipamentos informáticos. \\
& Carência de locais apropriados para a utilização dos recursos. \\
\hline Wheeler, Gordon-Brown, & Falta de conhecimentos/formação dos SIG por parte dos professores. \\
Peterson, \& Ward (2010) & Falta de tempo para que os professores desenvolvam competências geográficas \\
& através das TIG. \\
\hline Antunes (2007) & Desconhecimento das TIG e do potencial que as tecnologias suportam. \\
& $\begin{array}{l}\text { Insegurança na integração das tecnologias e da informática nas atividades le- } \\
\text { tivas. }\end{array}$ \\
\hline Kerski (2003) & Falta de tempo para incluir os SIG nas dinâmicas letivas. \\
& Complexidade do software. \\
& Custos de hardware e software. \\
\hline
\end{tabular}

como recursos didáticos. Um estudo realizado por Höhnle, Schubert e Uphues (2011) aferiu que, na perspetiva dos professores alemães, a maior barreira à utilização destas tecnologias foca-se na falta de formação docente, em TIG, e na prática recorrente da mesma. Este impedimento é igualmente referido por Hong (2014) e Wheeler et al. (2010) nos seus estudos, corroborando com a ideia de que a divulgação e, sobretudo, a aplicação destas ferramentas na formação docente é iminente. Através do exercício e da experiência com TIG, os futuros professores não só reconhecerão as potencialidades destas tecnologias na criação de momentos de aprendizagens verdadeiramente significativos, como sentir-se-ão seguros em incluí-las nas suas práxis. Desta forma, será possível mitigar alguns dos bloqueios à utilização das TIG e fomentar o enquadramento das mesmas no processo de aprendizagem dos futuros docentes e dos estudantes.

\section{Atlas geográfico de África: a transformação de ideias em realidade com as TIG}

Se atualmente se defende que o sistema de ensino se deve basear no construtivismo, segundo o qual os estudantes assumem um papel protagonizado na co-contrução do conhecimento (Becker, 2012), então, do mesmo modo, as instituições de ensino superior de educação devem fomentar, nos processos de profissionalização dos futuros professores, abordagens igualmente construtivistas.

Com o preceito de desenvolver a prática de utilização de ferramentas educacionais pioneiras, designadamente as TIG, alicerçada numa metodologia participativa, desenvolveu-se o projeto "Atlas de África". Proposto pelo docente da unidade curricular de Didática da Geografia, o projeto concretizou-se ao longo de um semestre.

O projeto desenvolveu-se em três fases principais (Figura 1). Na primeira fase, referente à recolha de informação, as estudantes inscritas na unidade curricular procederam ao levantamento dos dados relativos a quatro/cinco países.

Para esse efeito, várias ferramentas TIC e TIG foram utilizadas (ex: Google Earth, Google Maps), ora para recolha de informações estatísticas sobre a Geografia Física, Demografia, Economia e História/Cultura, ora para a recolha de coordenadas geográficas para posterior geolocalização das entidades geográficas. As informações coligidas foram

inseridas, de forma colaborativa, numa base de dados, em formato excel, permitindo, na segunda fase, tratar e analisar a informação obtida nas várias fontes. Através da tecnologia Esri, Arcmap, as estudantes procederam ao tratamento de uma parte da informação e, consequentemente, à produção de representações cartográficas. Parte do tratamento da informação foi realizada no excel, nomeadamente para a construção dos gráficos. Na última fase procedeu-se à organização e apresentação dos dados geográficos. 


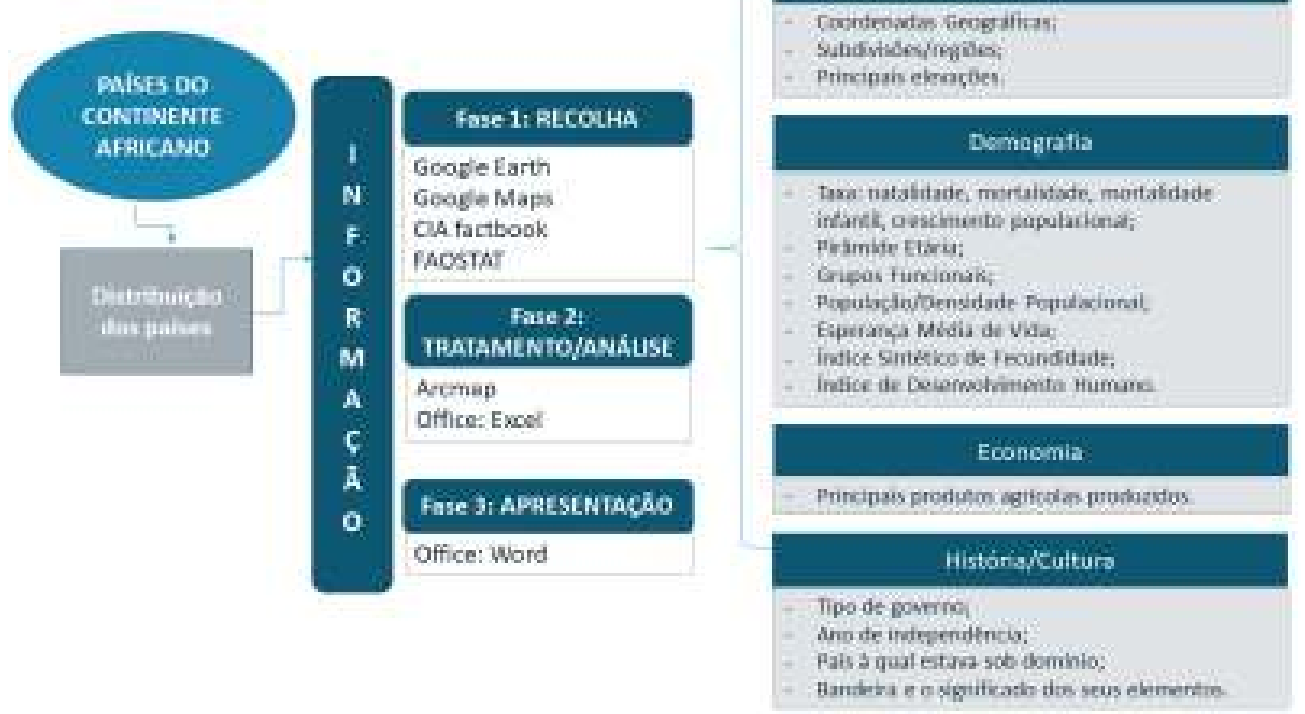

Figura 1: Etapas da investigação.

Deste modo, foram trabalhadas diversas competências desde a pesquisa científica de dados geográficas, através das fontes oficiais, à construção e manuseamento de bases de dados, tratamento da informação gráfica e cartográfica e aprofundamento do trabalho colaborativo.

\section{As TIG na sala de aula: resultados de um projeto interdisciplinar}

"Colocaram nas nossas mãos ferramentas com asas, com elas queremos abrir novos horizontes" (L.)

O projeto desenvolvido, que resultou na publicação de um atlas geográfico do continente africano (Figura 2), foi um desafio para as estudantes, futuras professoras, na medida em que manusearam, pela primeira vez, múltiplas ferramentas geoespaciais.
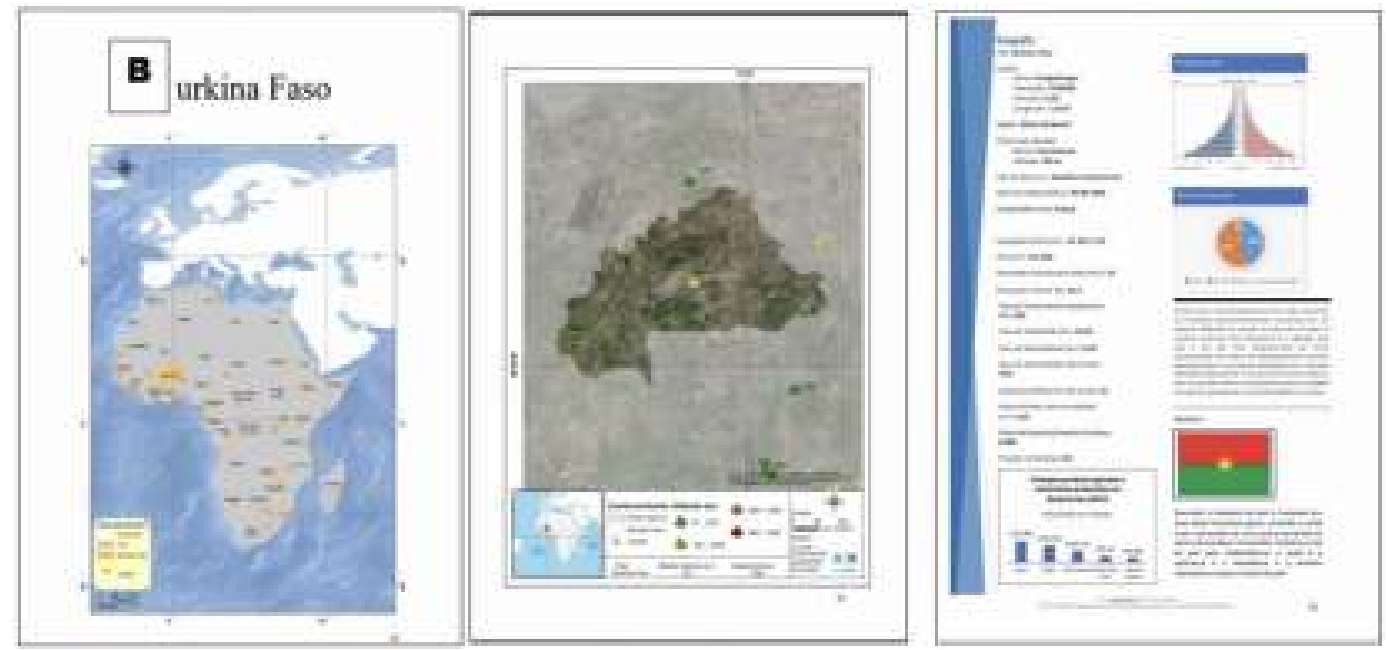

Figura 2: Layout do atlas - Burkina Faso.

Embora se tenha percecionado que o primeiro contacto com as TIG tenha sido encarado, por algumas estudantes, mais apreensivas com a tecnologia, como uma tarefa complexa, igualmente se 
constatou que, no final do projeto, as futuras profissionais revelaram destreza na sua utilização e reconheceram o potencial das mesmas na área educacional.

Na publicação "Viagem Geográfica por África: uma aplicação didática com as TIG" (2016) as estudantes expuseram as suas opiniões sobre o trabalho desenvolvido e sobre os benefícios das TIG no processo de ensino/aprendizagem, nomeadamente para o desenvolvimento de múltiplas competências essenciais para as práticas futuras. Como descreve uma das estudantes, "foi possível desenvolver competências essenciais e fundamentais, para abrir os horizontes daqueles que vão embarcar connosco durante longas viagens" (estudante A). Do mesmo modo, é consentido por estas que as TIG promove(ra)m a co-contrução do conhecimento e estimula(ra)m o pensamento crítico:

a descoberta de novas formas de promover o ensino e a aprendizagem da Geografia foi o ponto fulcral deste projeto. Poder aliar as potencialidades das TIG às aulas de Geografia e, deste modo, permitir que os alunos se envolvam ativamente na construção do seu próprio conhecimento e que desenvolvam o pensamento crítico é, sem dúvida, uma mais-valia. Ao longo desta experiência nós próprias tivemos oportunidade de nos aproximar das TIG e de, na primeira pessoa, compreendermos a sua importância no processo de ensino aprendizagem (estudante B).

As contrariedades sentidas pelas futuras profissionais de educação, ao longo do percurso, foram erradicadas pelo trabalho colaborativo existente entre as estudantes e o docente da unidade curricular, que orientou e auxiliou os vários elementos nos momentos de maior dificuldade. A utilização das Geotecnologias ao longo do projeto mobilizou momentos de partilha de incertezas e dúvidas, mas também de conhecimentos e de experiências, "partilhamos saberes" (estudante C), assim como impulsionou oportunidades de discussão de ideias e envolvimento das estudantes, contribuindo para a intensificação do empenho e motivação.

$\mathrm{Na}$ ótica das estudantes, a manipulação das Geotecnologias admite a exploração e compreensão do espaço geográfico, extrapolando os limites da sala de aula, através de viagens pelo mundo, nos contextos formais de aprendizagem:

vejo os SIG como um transcender da realidade que permite compreendê-la verdadeiramente.

Tal como um livro, leva-nos para outros lugares, sem sair do lugar. Eleva-nos para lá da estratosfera, sem sair da troposfera. E a elaboração deste altas, levou-nos a África, sem sair da sala de aula (estudante D).

Esta nova possibilidade de viajar admite que sujeitos provenientes de contextos mais desfavorecidos tenham a possibilidade de conhecer lugares que de outra forma não poderiam conhecer, quebrando as barreiras de infoexclusão. Por outro lado, permitiu que as estudantes explorassem, em diferentes escalas, a realidade geográfica de cada país "as TIG e os SIG permitiram o acesso, através de programas, funcionalidades e instrumentos tecnológicos, à realidade geográfica de todos os países" (estudante D).

A recolha de dados sobre os vários países ao nível da História, Economia, Demografia e Geografia Física evidenciou que as TIG potenciam a criação de pontes entre as várias áreas de estudo, promovendo uma aprendizagem interdisciplinar, facilitando a compreensão da relação entre factos e fenómenos, que estudados de forma estanque, seriam difíceis de perceber.

\section{Considerações finais}

Num mundo global, impactante, é iminente a intervenção e participação ativa dos estudantes. A revolução tecnológica exige, assim, que a escola acompanhe as modificações sociais, alterando os paradigmas de ensino. As instituições educativas de ensino básico, secundário e superior têm de deixar de olhar para as tecnologias emergentes como uma advertência e contemplá-las como uma oportunidade de munir as gerações mais jovens de competências essenciais para o futuro mercado de trabalho.

O projeto apresentado neste artigo revela como as ferramentas geográficas podem, facilmente, ser apreendidas, mesmo em contextos onde os conhecimentos prévios são muitos escassos. Através de um projeto, como aquele que aqui foi apresentado, pode-se fomentar o trabalho colaborativo, aumentando 
a responsabilidade dos estudantes, e aumentar os seus aspetos motivacionais. A concretização do trabalho colaborativo do grupo sob a forma de publicação revelou-se também como um motor para que as estudantes empregassem maior responsabilidade nas pesquisas científicas que realizaram e no diálogo constante entre elas.

Neste sentido, revelou-se muito pertinente a formação das futuras profissionais na utilização das TIG para apreender um conjunto de conceitos chave de Geografia. Mais do que formar para a utilização dos SIG e das Geotecnologias revela-se fundamental fomentar a aprendizagem através dos SIG. Mais importante do que saber manipular estas ferramentas é perceber como se pode usar as TIG para desenvolver a aprendizagem de outros domínios que, na maior parte dos casos, extravasa a Geografia e os seus indicadores de base. A elaboração do atlas geográfico, através das TIG e do manuseamento de indicadores de base, tratados em base de dados, permitiu também desenvolver um conjunto de competências relacionadas com as TIC. Os autores consideram muito positivo o trabalho colaborativo para a aquisição de competências transversais de uma forma mais empenhada e frutífera.

\section{Referências}

Antunes, S. (2007). Integração dos SIG/WEBSIG na formação inicial de docentes do $1 .{ }^{\circ}$ ciclo do ensino básico. Dissertação de mestrado, ISEGI, Universidade Nova de Lisboa, Lisboa, Portugal.

Azevedo, L. (2016). As potencialidades das tecnologias de informação geográfica no processo de ensino e aprendizagem dos alunos do 1. ${ }^{\circ}$ e do 2. ${ }^{\circ} \mathrm{CEB}$. Dissertação de mestrado, Escola Superior de Educação de Paula Frassinetti, Porto, Portugal.

Becker, B. (2012). Educação e construção do conhecimento. Porto Alegre: Penso.

Bednarz, S. W., \& Schee, J. (2006). Europe and the United States: the implementation of geographic information systems in secondary education in two contexts. Technology, Pedagogy and Education, 15(2), 191-205

Demirci, A. (2009). How do teachers approach new technologies: geography teachers' attitudes towards geographic information systems (GIS). European Journal of Educational Studies, 1(1), 57-67.

Erskine, M. A., Dawn, G., \& Karimi, J. (2016). Perceptions and attitudes toward online mapping services. Journal of Computer Information Systems, 5(2), 175-184.

Gomes, N. (2006). Potencial didáctico dos sistemas de informação geográficos no ensino da geografia: aplicação 3. ${ }^{\circ}$ ciclo do ensino básico. Dissertação de mestrado, ISEGI, Universidade Nova de Lisboa, Portugal.

Goodchild, F. (2009). Geographic information systems and science: today and tomorrow. Procedia Earth and Planetary Science, 1(1), 1037-1043.

Healey, M. (2005). Linking research and teaching to benefit student learning. Journal of Geography in Higher Education, 29(2), 183-201.

Hong, J. (2014). Promoting teacher adoption of GIS: using teacher-centered and teacher-friendly design. Journal of Geography, 113(4), 139-150.

Kopcha, T. J., Otumfuor, B. A., \& Wang, L. (2015). Effects of spatial ability: gender differences, and pictorial training on children using 2-D and $3-\mathrm{D}$ environments to recall landmark locations from memory. Journal of Research on Technology in Education, 47(1), 1-20.

Prensky, M. (2001). Digital natives, digital immigrants part 1. On the horizon, 9(5), 1-6.

Ribeiro, V. (Coord.). (2016). Viagem geográfica por África: uma aplicação didática com as TIG. Acedido de http://repositorio.esepf.pt/handle/20.500.11796/2268 
Ribeiro, V., Azevedo, L., \& Osório, A., 2016. Promoting a spatial perspective on primary students through geotechnologies. In. L. Gómez Chova, A. López Martínez and I. Candel Torres (Eds.) Proceedings of the 8th International Conference on Education and New Learning Technologies-EDULEARN16 (pp. 6455-6459). Barcelona: IATED Academy.

Ribeiro, V., Remoaldo, P., \& Gutiérrez, J. (2015). Measuring the accessibility of bus stops for elderly people: the effects of slope and walking speeds. In A. Melhorado-Condeço, A. Regianni, \& J. Gutiérrez (Orgs.) Accessibility and spatial interaction (pp. 315-327). Londres: Edward Elgar Publishing.

Santos, Â. (2010). Os sistemas de informação geográfica no ensino da geografia: aplicação a uma turma do $3^{\circ}$ ciclo do ensino básico. Dissertação de mestrado, ISEGI, Universidade Nova de Lisboa, Lisboa, Portugal.

Schultz, B., Kerski, J., \& Patterson, T. (2008). The use of virtual globes as a spatial teaching tool with suggestions for metadata standards. Journal of geography, 107(1), 27-34.

Spronken-Smith, R., \& Walker, R. (2010). Can inquiry-based learning strengthen the links between teaching and disciplinary research? Studies in Higher Education, 35(6), 723-740.

Van der Schee, J. (2003). New media will accelerate the renewal of geographic education. In R. Gerber International handbook on geographical education (pp. 205- 213). Dordrecht: Kluwer.

Wheeler, P., Gordon-Brown, L., Peterson, J., \& Ward, M. (2010). Geographical information systems in victorian secondary schools: current constraints and opportunities. International Research in Geographical and Environmental Education, 19(2), 155-170.

Wickson, F., Carew, A., \& Russell, AW. (2006). Transdisciplinary research: characteristics, quandaries and quality. Futures, 38(9), 1046-1059 